\title{
Lung-Digit Syndrome Related to an Adenosquamous Feline Lung Carcinoma
}

\author{
Felipe Noleto de Paiva', Max Ferreira de Andrade², Dayane Caicó Collares Araújo', Rafaela da Silva Goes', \\ Thiago Souza Costa', Vivian de Assunção Nogueira Carvalho' \& Julio Israel Fernandes ${ }^{1}$
}

\begin{abstract}
Background: The lung-digit syndrome is a rare syndrome characterized by the presence of a primary pulmonar neoplasia manifesting metastasis to the digit, occurring exclusively in felines. The diagnosis is based on the clinical signs, associated with radiographic and histopathological exams. There are no therapeutic protocols well-established, and surgical excision is considered controversial due to high recurrence and metastasis rates. The prognosis is considered poor, with low survival rates. The aim of this paper is to report a case of lung-digit syndrome attended in Rio de Janeiro.

Case: A 12-year-old female cat, no defined race, was attend with the complaint of weight loss and injury in the right thoracic limb, already having histopathological diagnosis of squamous differentiation adenocarcinoma through biopsy. Physical examination showed no other clinical signs at first, including no signs of respiratory disease. Laboratory and imaging exams were performed, and the radiographic examination showed alterations in the pulmonary parenchyma showing a nodular area of increased radiographic density. Followed up by the manifestation of breathing noise at rest, as the first respiratory signs. The association of the clinical evaluation, medical history, and histopathological report from the limb lesion, lead to the suspicion diagnostic of lung-digit syndrome. The owners decide for the palliative treatment with chemotherapy, using carboplatin and prednisolone. Only the first session was performed, with the animal being euthanized due to clinical worsening 48 days after the initial manifestation of clinical signs. The diagnosis was confirmed by post mortem exams, and the pulmonary nodule were diagnosed as adenocarcinoma with squamous differentiation in concordance with the limb lesion diagnosis.

Discussion: The lung-digit syndrome is still poorly studied in the veterinary medicine, with few reports published. Epidemiological data shows major incidence in elderly cats, with no racial or sexual predisposition, occurring in a 12-year-old cat in the present case. There are few theorys to explain the etiopathogenesis of the syndrome, however, none have been fully comproved. The clinical symptomatology varies from asymptomatic animals to those manifesting nonspecific systemic signs and respiratory signs. In this case the animal initially manifested only the presence of the digit injury and weight loss, with respiratory signs manifesting later. The digital lesion local signs usually include swelling, ulceration, purulent discharge, nail loss, and pain, occurring more frequently in the thoracic limbs, involving weight-bearing digits, as in the present report that occurred in the right thoracic limb, with involvement of the first and third digits. Radiographic findings may help in the diagnosis suspicion, having the confirmation by histopathological examination. The most frequent histological types involved in the syndrome are the adenocarcinoma, bronchoalveolar carcinoma, squamous cell carcinoma and adenosquamous carcinoma. The squamous-differentiated adenocarcinoma or adenosquamous carcinoma, as described in the histopathological report in the present case, is the least frequent. The treatment protocol is not well-established with no proven effective treatment. Surgical excision is not recomended as a palliative method, and the chemotherapy and radiotherapy sucess rates are still unknown. The prognosis is considered extremely unfavorable and early diagnosis represents the best attempt to control the disease.
\end{abstract}

Keywords: neoplasia, pulmonary, digital metastasis, domestic cat. 


\section{INTRODUCTION}

Primary lung neoplasms are considered rare in domestic animals [6] in contrast to human medicine [23]. The incidence is low in domestic cats [5], accounting for $0.69 \%$ of cases of feline neoplasms [7], as shown by Barr et al. [3] that reported 17 cases over a period of 16 years. Etiology is unknown, although there are indications of the influence of pollutants and air quality [23].

Clinical signs include respiratory and nonspecific signs $[1,2,5,12,20]$. Asymptomatic animals represent the minoriry of cases, where neoplasia becomes an incidental finding [2,22]. Biological behavior is considered aggressive [6], with metastasis rates of $60 \%$ to $80 \%[2,7,12]$. Most frequently location is intrapulmonary [7,12], followed by lymph nodes, liver, spleen, kidneys, and intestines [7,22] and less frequently ocular choroid, bones, musculature, salivary gland and skin $[9,15,17,22,24]$. Digit metastases are considered frequent among extrapulmonary involvement, accounting for up to $17.9 \%$ of all metastasis cases [7].

The lung-digit syndrome is defined as the manifestation of a primary pulmonary neoplasm with metastasis specifically to the digits [5]. This syndrome is described exclusively in felines and is not recognized in other domestic animals [18,19]. However, there are similar reports in human medicine, in case of a 62-year-old man manifesting a primary pulmonary squamous cell carcinoma with toe metastasis [25]. The aim of the present study is to report the clinical approach to a case of the syndrome.

\section{CASE}

A 12-year-old, female cat, mixed-breed, sexually intact, not tested for the feline immunodeficiency vírus (FIV) and feline leukemia vírus (FeLV), attended to the Oncology Service of the Veterinary Hospital of the Rural Federal University of Rio de Janeiro, with complaint of injury in the digits of the right thoracic limb Figure 1) and recent weight loss, showing a one month evolution.

As reported by the owners, the animal had already undergone external veterinary care, in which was performed an excisional biopsy, with histopathological diagnosis of squamous differentiation adenocarcinoma. Concomitantly, fungal culture was performed due to sporotrichosis suspiccion, and the result was negative.

On physical examination, vital parameters of heart rate, respiratory rate, mucosal color and rectal temperature were within the normal range for the specie. In the digit it was observed a lesion in the interdigital region, presenting ulcerated, adhered, firm and hyperemic, and the animal manifested pain on palpation. Laboratory tests of blood count and serum biochemical profile with analysis of albumin, alanine aminotransferase (ALT), gamma glutamyl transpeptidase (GGT), creatinine and urea, and immunochromatographic examination for FIV and FeLV were requested. The results showed no noteworthy change. FIV and FeLV tests were negative.

Subsequently, abdominal ultrasonography, and radiography from the chest and the affected limb were performed. Ultrasound examination showed no

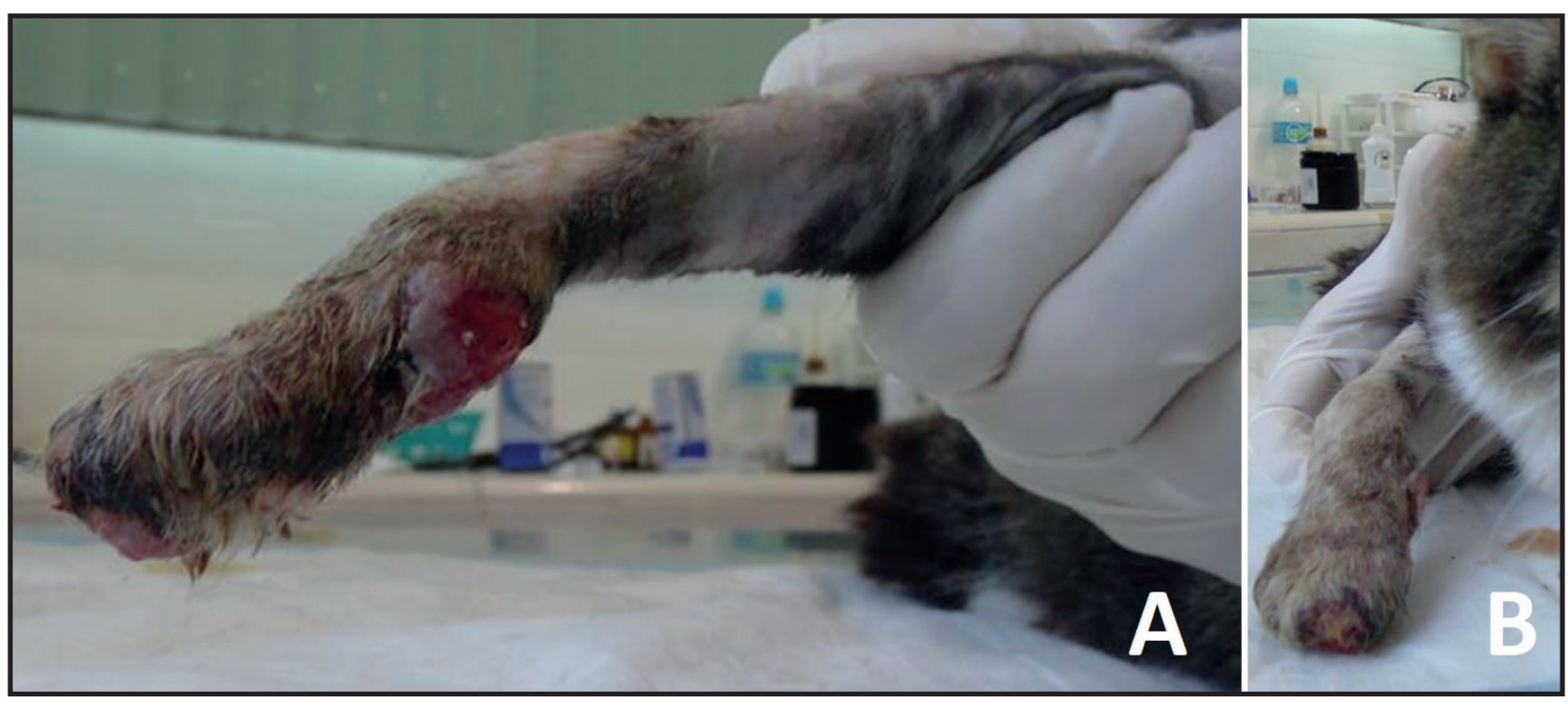

Figure 1. A 12-year-old female cat, showing ulcerated, hyperemic and exudative limb lesion. A- Limb injury, lateral view. B- Limb injury, frontal view. 
noticeable changes. Radiography showed alteration in the pulmonary parenchyma with the presence of a diffuse infiltrate interstitial pattern, with an area of increased radiographic density, measuring $3.0 \mathrm{~cm}$ in diameter located in topography of left diaphragmatic lobe (Figure 2). A smaller nodule was observed, but was later recognized as a radiographic artifact. In the digit evaluation, a slight loss of radiographic density was observed in distal phalanges of the first and third digits, showing osteopenia suggestive of neoplastic infiltration. Upon returning for reassessment, the animal began to show breathing noise at rest.

$\mathrm{Na}$ initial diagnostic of lung-digit syndrome was established, considering pulmonary neoplasia as primary focus and digit injury as metastatic focus. According to the TNM staging system, by the World Health Organization (WHO), the tumor was considered T1, a solitary lung tumor; N0, without nodal involvement; M1 with distant metastasis, adding T1N0M1, configuring stage four disease. Palliative chemotherapeutic treatment was recommended, with Carboplatin (B-Platin $\left.{ }^{\circledR}\right)^{1} 150 \mathrm{mg} / \mathrm{m}^{2}$ dose, administered intravenous every 21 days, totaling 6 cycles, associated with Prednisolone (Prediderm $\left.{ }^{\circledR}\right)^{2} 1 \mathrm{mg} / \mathrm{kg}$ dose, orally, every $12 \mathrm{~h}$, throughout the treatment.

The protocol started with no side effects within the $48 \mathrm{~h}$ after the application, however the animal suffered a drastic worsening in the general clinical condition, manifesting acute and unproductive cough, prostration, inappetence and continuous weight loss.
The digit lesion also worsened considerably manifesting swelling and ulceration with active bleeding. Facing the low response to treatment and the poor prognosis, the owners choosen for eutanásia.

In necropsy examination, through macroscopic cutaneous evaluation, an exudative and ulcerated lesion involving the third and four digits was described. In the internal evaluation, the presence of a single nodule in the left diaphragmatic lobe measuring $5 \times 3 \times 1.5 \mathrm{~cm}$ was observed (Figure 3), with no significant changes in other organs.

In the microscopic evaluation of the pulmonary lesion, it was described multifocal areas of epithelial cell proliferation, organized in bundles or islands, of glandular aspect and polyhedral cell morphology, with eosinophilic cytoplasm, round and basophilic nucleus, evident nucleoli, anisocytosis and anisocariosis. The diagnosis was a pulmonary adenocarcinoma with areas of squamous differentiation. Microscopic evaluation of the digit lesion was impaired by marked fibrosis and autolysis, but it was possible to observe cellularity with a pattern suggestive of adenocarcinoma, confirming the lung-digit syndrome diagnosis.

\section{DISCUSSION}

Epidemiological studies regarding lung-digit syndrome are scarce in the literature, however, data report that more than $80 \%$ of digit carcinomas are primary tumor metastases [18]. The epidemiological profile of this animals tend to be similar to the profile

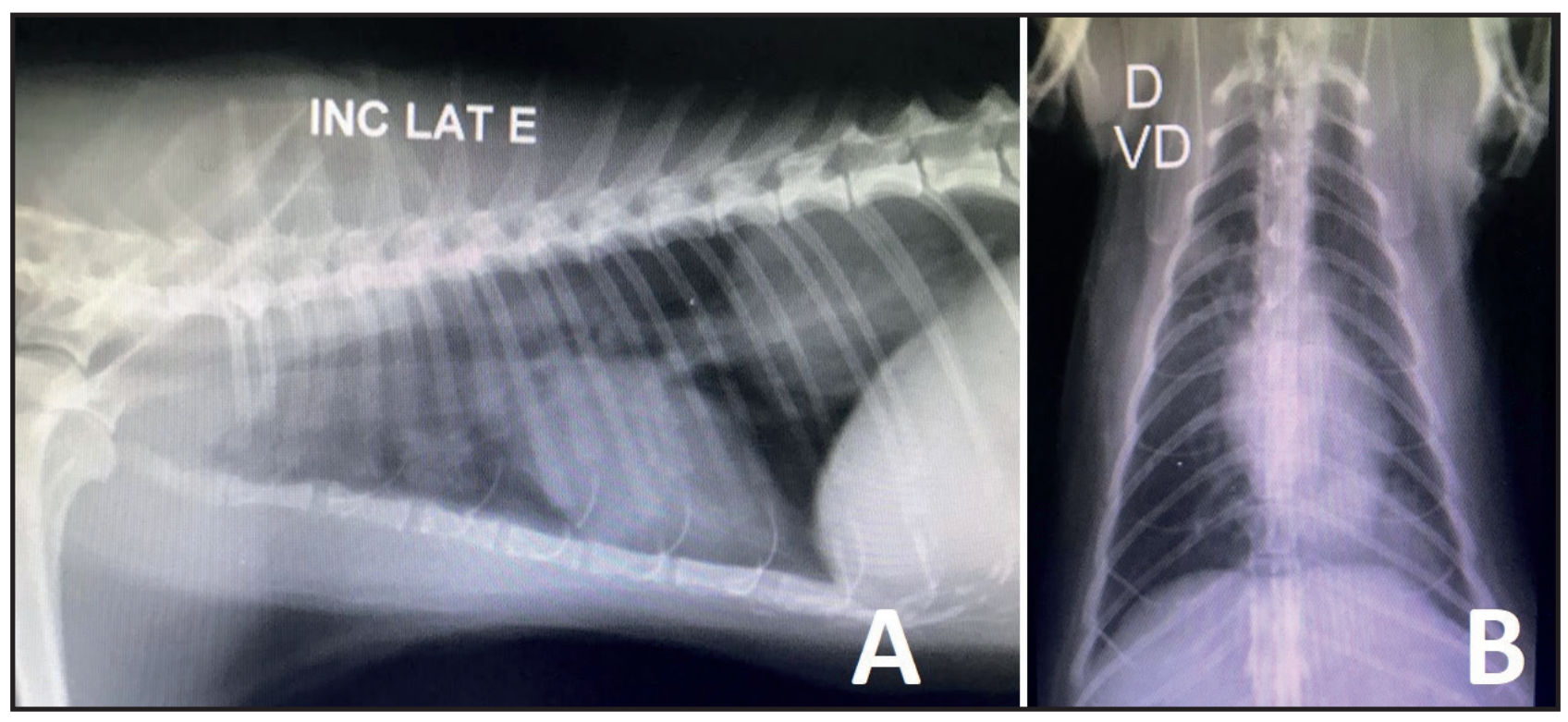

Figure 2. Radiographic projections of 12-year-old female cat thorax with lung-digit syndrome showing focal areas of increased radiographic density. A- Left lateral-lateral projection. B- Ventral-dorsal projection. 


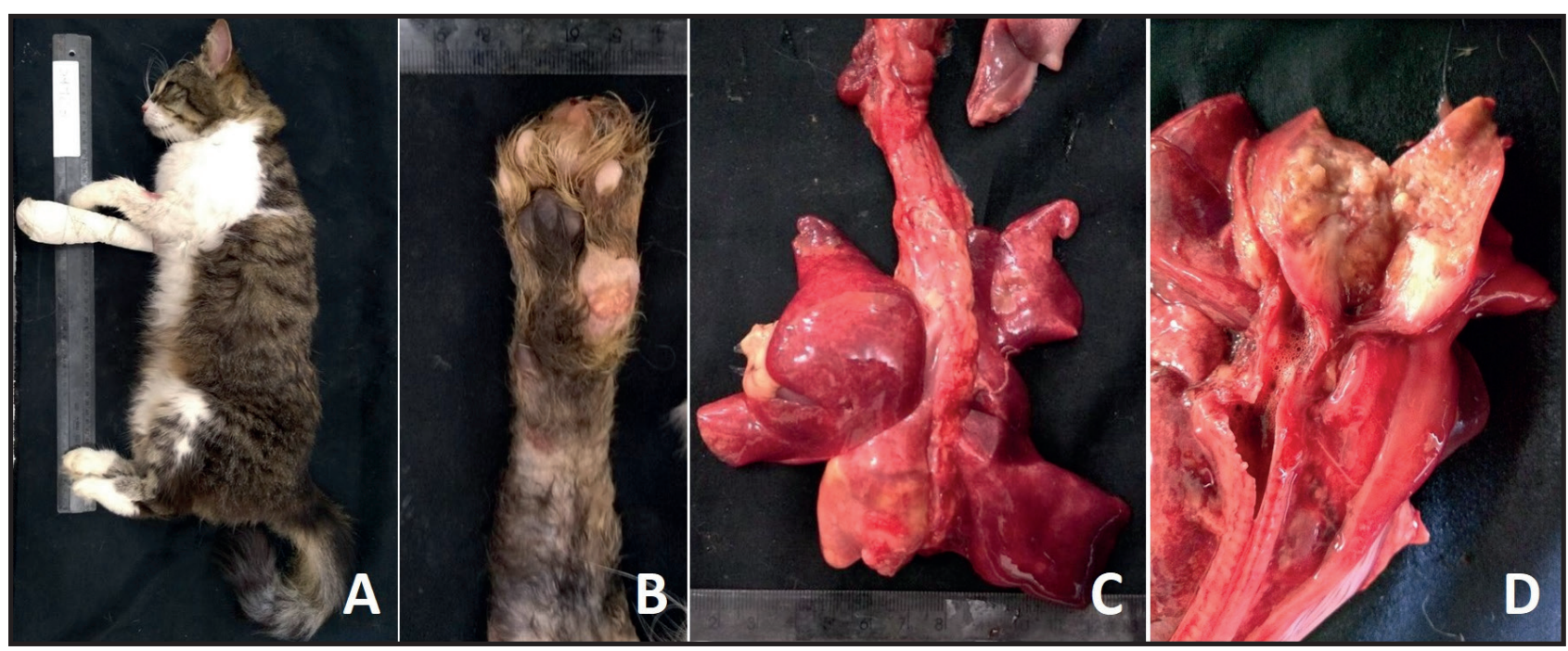

Figure 3. Evaluation of a 12-year-old female cat at necroscopic examination. A- General body state. B- Exudative and ulcerated lesion involving third and fourth digits. C- General evaluation of the lungs. D- Nodule in left diaphragmatic lobe measuring $5 \times 3 \times 1.5 \mathrm{~cm}$ affecting the entire lobe.

of the ones affected by primary pulmonary neoplas$\mathrm{ms}$, that includes animals ranging in age from 4 to 20 years, on an average of 12 years old, as in the present report of a 12-year-old feline. Isolated case reports of the syndrome mainly describe animals over 10 years of age [14,16,26,28], with few reports below 10 years old $[8,22]$. There is no reported racial or sexual predisposition $[11,18]$. In this case, the animal tested negative for FIV and FeLV, although no correlation between this neoplasms and the viruses has been described [12].

The etiopathogenesis of lung-digit syndrome is not well established, and there are two main theories that explain the manifestation. The first is related to the mechanism of dissemination, most commonly occurring through the hematogenous pathway, which added to the extensive vascularization present in the feline digital region, could predispose the establishment of metastasis in the region [18]. A second theory states that the predilection of metastasis to the digital region exists due to the specific characteristics present in the tissue microenvironment, related to growth factors, chemotactic agents and endothelial cell adhesion molecules that predispose to involvement [26]. Further studies are needed to substantiate the proposed hypotheses.

Pulmonary neoplasms are often associated with nonspecific signs and may be associated with respiratory signs [1-3,5,12,13,20,22]. In specific cases of lung-digit syndrome, the initial complaint often consists of digital injury and local signs [8,11,14,21,22,26-28] and may be accompanied by systemic signs of weight loss, inappetence, anorexia, lethargy and behavioral changes $[14,16,28]$.

Only the minority of cases present respiratory signs at the time of initial assessment [21], with some cases of pre-existing respiratory disease [11]. In the present report, the initial complaint presented by the pet owners was the presence of the digit injury associated with recent weight loss, with respiratory signs later manifesting with breathing noise at rest. The initial manifestation of digital lesion without respiratory manifestation is in agreement with that observed by Gottfried et al. [11] who evaluated 36 cases of the syndrome, in which none of the animals showed respiratory signs at the first evaluation. As the disease progresses, respiratory symptoms may occur [16,22], although in many cases the animal dies before such manifestation [8,10,14,26,28].

In the digital lesion, the most commonly signs are swelling, ulceration, purulent discharge, nail loss, and pain $[11,21,22,26-28]$, similar to the signs observed in the present report. Lesion distribution occurs most frequently in the digits of the thoracic limbs against the pelvic ones, and usually involves primary weight-bearing digits [11]. It commonly affects the digits, with less involvement in the nail bed [27]. Cases of digit involvement in more than one limbs are rare [11]. The location recorded in the case occurred in the right thoracic limb, with involvement of the first and third digits, considered supporting digits, corroborating with the described in the literature. 
Radiographic imaging of the limb may reveal osteolysis as the most common finding $[10,21,22,26,28]$, besides bone proliferation with or without joint involvement $[10,21,26]$. The patient's digital radiographic image showed slight density loss compatible with osteolysis as described.

Among the primary pulmonary neoplasms, the most frequent histological types are adenocarcinoma, bronchoalveolar carcinoma, squamous cell carcinoma and adenosquamous carcinoma respectively $[1-3,5,7,12,13,20,23]$. In cases of lung-digit syndrome, adenocarcinoma also occurs more frequently $[10,14,22,27,28]$, with fewer reported cases of bronchoalveolar carcinoma [11,28] and squamous cell carcinoma $[8,26]$. Cases of squamous-differentiated adenocarcinoma or adenosquamous carcinoma, as described in the histopathological report, are the least frequent.

Radiography exam can identify or suggest the presence of lung câncer [10], although computed tomography is considered the most accurate examination for such cases $[1,28]$. In the case described, the radiographic examination showed the presence of the mass. The classic presentation is the presence of a solitary and circumscribed nodule or mass $[8,10,11,14,26,27]$, occurring less frequently as multiple lesions $[11,21]$. In cases of effusion, the lesions may be obscured [21,22]. Radiographic presentation of the patient occurred in a clear pulmonary nodule without effusion. The radiographic pattern cannot predict the tumor types [3].

The clinical manifestation added to the radiographic findings are suggestive to the manifestation of the syndrome [10], although the histopathological evaluation of both lung mass and digit injury is necessary for the confirmatory diagnosis [10,27]. In the reported case, the animal already had a previous diagnosis of adenosquamous carcinoma performed after biopsy of the limb lesion, and had the diagnosis of pulmonary mass in post mortem examination, confirming the diagnosis. Cytological examination is not considered standard for diagnosis of lung câncer [10,20,22]. In the present report the cytology exam was not performed as the animal already had a histopathological exam by the time of the first consultation. In many cases the final diagnosis can only be obtained by post mortem evaluation on necropsy exam $[8,16,21,28]$, as in the described report, in which the final diagnosis was obteined in post mortem evaluation.
The laboratory tests have poor diagnostic value in cases of lung-digit syndrome [29], without marked changes in blood count, serum biochemistry and urinalysis $[10,11,14,26]$. Isolated cases of anemia, leukocytosis, azotemia have been reported [10,28]. In the present report, no changes in laboratory tests were observed.

Cases of the syndrome are often marked by the presence of concomitant pulmonary metastases in other sites $[8,10,11,18,22,27,28]$. The most common location is the musculature [10,18,22,27,28], also occurring in other regions as the skin, lymph nodes, pleura, liver, spleen, kidneys, eyes, bones, salivary gland, intestines, brain and heart [8,10,11,18,22,27,28]. In the present report there was no metastasis detected in other organs until the moment of diagnosis or in post mortem diagnosis, characterizing a case, of solitary digital metastasis.

There is no well-established terapeutic protocol for cases of lung-digit syndrome, and no proven effective treatment [10,27]. Surgical excision may be performed for diagnostic purposes [11], but is not considered as a palliative method due to poor survival time, potential reduction in quality of life, and high rates of metastasis and relapse $[10,11,27]$. In the case presented, surgical excision was performed prior to the initial consultation, allowing the definitive diagnosis, although it was not possible to evaluate it real therapeutic value. Data about chemotherapy and radiotherapy are still scarce, without proven benefits [27]. In the report, the palliative chemotherapy treatment has been used as chosen by the owners. It was performed only one session of the carboplatin protocol associated with prednisolone, before the animal's death. The use of chemotherapy has been reported by Hanselman \& Hall [14] with an epirubicin protocol, showing no effective response, with death after two applications. Some authors report the use of symptomatic drug treatment, also with low clinical response [14,22,28].

The prognosis for the lung-digit syndrome is considered extremely unfavorable [10], with most cases reporting euthanasia without therapeutic intervention $[8,16,22,26]$ or after clinical worsening $[14,22,28]$, as occurred in the present case. The reported survival time ranges from 34 to 104 days according to Sugiyama et al. [27] and 12 to 122 days according to Gottfried et al. [11] with an average of 59 days $[11,27]$. Using surgical treatment associated 
with chemotherapy and suport treatment Hanselman $\&$ Hall [14] reached 42 days of survival time. In the present report, a 23 day survival time was reached, corroborating the data described in the literature. More studies are needed about the syndrome in order to establish protocols and define the most effective therapy.

In conclusion, given the extremely unfavorable prognosis and the low efficacy associated with therapeutic modalities available in veterinary medicine, early diagnosis assumes great importance in the attempt to control the disease, although even in this cases te eutanasia is still an option.

\section{MANUFACTURERS}

${ }^{1}$ Blausiegel Indústria e Comércio Ltda. Itapecerica da Serra, SP, Brazil.

${ }^{2}$ Ourofino Saúde Animal. Cravinhos, SP, Brazil.

Declaration of interest. The authors report no conflicts of interest. The authors alone are responsible for the content and writing of the paper.

\section{REFERENCES}

1 Aarsvold S., Reetz J.A., Reichle J. K., Jones I.D., Lamb C.R., Evola M.G., Keyerleber M.A. \& Marolf A.J. 2015. Computed tomographic findings in 57 cats with primary pulmonary neoplasia. Veterinary Radiology \& Ultrasound. 56(3): 272-277.

2 Albert L., Majó N., Pastor J. \& Planellas M. 2012. Carcinoma pulmonar primario en gatos: 10 casos (1998-2011). Clínica Veterinaria de Pequeños Animales. 32(4): 247-253.

3 Barr F., Gruffydd-Jones T.J., Brown P.J. \& Gibbs C. 1987. Primary lung tumours in the cat. Journal of Small Animal Practice. 28(12): 1115-1125.

5 Costa F.V.A., Souza H.J.M., Cunha S.C.S. \& Corgozinho K.B. 2017. Oncologia Felina. Rio de Janeiro: L.F. Livros, 640p.

6 Dalek C.R. \& Nardi A.B. 2016. Oncologia em Cães e Gatos. 2.ed. São Paulo: Roca, 746p.

7 D'Costa S., Yoon B.I., Kim D.Y., Motsinger-Reif A.A., Williams M. \& Kim Y. 2012. Morphologic and Molecular Analysis of 39 Spontaneous Feline Pulmonary Carcinomas. Veterinary Pathology. 49(6): 971-978.

8 Dhaliwal R.S. \& Kufuor-Mensah E. 2007. Metastatic squamous cell carcinoma in a cat. Journal of Feline Medicine and Surgery. 9(1): 61-66.

9 Favrot C. \& Rubiales F.D. 2005. Cutaneous metastases of a bronchial adenocarcinoma in a cat. Veterinary Dermatology. 16(3): 183-186.

10 Goldfinch N. \& Argyle D. 2012. Feline lung-digit syndrome: Unusual metastatic patterns of primary lung tumours in cats. Journal of Feline Medicine and Surgery. 14(3): 202-208.

11 Gottfried S.D., Popovitch C.A., Goldschmidt M.H. \& Schelling C. 2000. Metastatic digital carcinoma in cat: a retrospective study of 36 cats (1992-1998). Journal of the American Animal Hospital Association. 36(6): 501-509.

12 Hahn K.A. \& Mcentee M.F. 1997. Primary lung tumors in cats: 86 cases (1979-1994). Journal of the American Animal Hospital Association. 211(10): 1257-1260.

13 Hahn K.A. \& Mcentee M.F. 1998. Prognosis factors for survival in cats after removal of a primary lung tumor: 21 cases (1979-1994). Veterinary Surgery. 27(4): 307-311.

14 Hanselman B.A. \& Hall J.A. 2004. Digital metastasis from a primary bronchogenic carcinoma. Canadian Veterinary Journal. 45(7): 614-616.

15 Heider H.J., Loesenbeck G., Heider E. \& Drommer W. 1997. Intraocular metastasis of bronchial carcinoma in a cat. Tierärztliche Praxis. 25(3): 271-274.

16 Jacobs T.M. \& Tomlinson M.J. 1997. The lung-digit syndrome in a cat. Feline Practice. 25(1): 31-36.

17 Langlais L.M., Gibson J., Taylor J.A. \& Caswell J.L. 2006. Pulmonary adenocarcinoma with metastasis to skeletal muscle in a cat. Canadian Veterinary Journal. 47(11): 1122-1123.

18 Linde-Sipman J.S. \& Ingh T.S. 1999. Primary and metastatic carcinomas in the digits of cats. Veterinary Quarterly. 22(6): 141-145.

19 Marino D.J., Matthiesen D.T., Stefanacci J.D. \& Moroff S.D. 1995. Evaluation of dogs with digit masses: 117 cases (1981-1991). Journal of the American Animal Hospital Association. 207(6): 726-728. 
F.N. Paiva, M.F. Andrade, D.C.C. Araújo, et al. 2020. Lung-Digit Syndrome Related to an Adenosquamous Feline Lung Carcinoma. Acta Scientiae Veterinariae. 48(Suppl 1): 515.

20 Maritato K.C., Schertel E.R., Kennedy S.C., Dudley R., Lamm C., Barnhart M. \& Kass P. 2014. Outcome and prognostic indicators in 20 cats with surgically treated primary lung tumors. Journal of Feline Medicine and Surgery. 16(12): 979-84.

21 May C. \& Newsholme S.J. 1989. Metastasis of feline pulmonary carcinoma presenting as multiple digital swelling. Journal of Small Animal Practice. 30(5): 302-310.

22 Moore A.S. \& Middleton D.J. 1982. Pulmonary adenocarcinoma in three cats with non respiratory signs only. Journal of Small Animal Practice. 23(9): 501-509.

23 Moulton J.E., Tscharner C.V. \& Schneider R. 1981. Classification of lung carcinomas in the dog and cat. Veterinary Pathology. 18(4): 513-528.

24 Nakanishi M., Kuwamura M., Ueno M., Yasuda K., Yamate J. \& Shimada T. 2003. Pulmonay adenocarcinoma with osteoblastic bone metastases in cat. Journal of Small Animal Practice. 44(10): 464-466.

25 Nicholson S., Mohamed I., Tahir A. \& Antunes G. 2011. Primary lung cancer presenting as metastasis to the big toe. International Journal of Surgery Case Reports. 2(5): 1-4.

26 Scott-Moncrieff J.C., Elliott G.S., Radovsky A. \& Blevins W.E. 1989. Pulmonary squamous cell carcinoma with multiple digital metastases in a cat. Journal of Small Animal Practice. 30(12): 696-699.

27 Sugiyama H., Maruo T., Shida T., Ishikawa T., Kanakubo K., Madarame H., Kayanuma H. \& Suganuma T. 2010. Clinical findings in lung-digit syndrome in five cats. Journal of Japan Veterinary Cancer Society. 1(1): 8-13.

28 Thrift E., Greenwell C., Turner A.L., Harvey A.M., Maher D. \& Malik R. 2017. Metastatic pulmonary carcinomas in cats ('feline lung-digit syndrome'): further variations on a theme. Journal of Feline Medicine and Surgery. 3(1): 1-8.

29 Withrow S.J. \& Vail D.M. 2007. Small Animal Clinical Oncology. 4th edn. St. Louis: Saunders Elsevier, 864p. 